\title{
Modelling the Botswana Pula/Us Dollar exchange rate using the Skewed generalized t (SGT) distributions
}

\author{
Wilson Moseki Thupeng \\ Department of Statistics, University of Botswana, Private Bag UB00705, Gaborone, Botswana
}

\begin{abstract}
The economy of Botswana heavily relies on mineral exports (mainly diamond exports), which are largely dependent on the exchange rate. And, the US Dollar is one of the most important currencies in the basket of currencies to which the Botswana Pula is pegged. Therefore, this paper seeks to empirically establish the baseline characteristics of the Botswana Pula (BWP) and the US Dollar (USD) exchange rate and to identify the most plausible probability distribution from the skewed generalized $t$ (SGT) family that can be used to model the log-returns of the daily BWP/USD exchange rates for the period January 2001 to December 2020. The SGT family is a highly versatile class of models that can capture the skewness and kleptokurticity that are inherent in financial time series. Four probability distributions are considered in this study: skewed $t$, skewed generalized error, generalized $t$ and skewed generalized $t$. The maximum likelihood approach is used to estimate the parameters of each model. Model comparison and selection are based on the Akaike information criterion (AIC) and Bayesian information criterion (BIC). The results of the study show that the daily BWP/USD exchange rate series is nonnormal, negatively skewed heavy-tailed. It is also found that, based on the values of both the AIC and BIC, the model that gives the best fit to the data is the skewed $t$, which is closely followed by the skewed generalized error distribution, while the generalized $t$ gives the worst fit.
\end{abstract}

Keywords: Pula/US Dollar exchange rate, log returns, Generalized t distribution, Skewed generalized error distribution, Skewed generalized $\mathrm{t}$ distribution, Skewed $\mathrm{t}$ distribution, skewness, kurtosis, maximum likelihood

\section{Introduction}

Botswana's economy remains heavily dependent on mineral exports (especially the diamond subsector), despite government's efforts to diversify the economy. Mining is, by some measures, the largest contributor to gross domestic product (GDP), generates the majority of export earnings and makes a major contribution to government revenues (African Development Bank, 2016). According to Sejoe, Sinha and Kahaka (2020), in the 2017/18 fiscal year, when combined, mineral revenues and customs and excise receipts accounted for 63 percent of total revenues during the period. This, the authors posit, indicates the importance of trade and how critical policy maker's decision on the exchange rate policy can influence the growth of the economy. The US Dollar is one of the most important currencies in the basket of currencies to which the Botswana Pula is pegged. For this reason, research on the Botswana Pula (BWP) and the US Dollar (USD) exchange rate using statistical methods, amongst others, by academics, regulators of financial institutions and investors has been gaining currency. The study by Sejoe et al. (2020) uses cointegration approach to investigate the Purchasing Power Parity (PPP) theory of the Pula/Rand and Pula/US Dollar exchange in Botswana for the period of 1976-2016. In the present paper, we contribute to the literature on the BWP/USD exchange rate by determining a probability model that adequately describes the BWP/USD exchange rate. This is a key step in the construction of market risk assessment tools like the Value-at-Risk (VaR). Further, as pointed out by Baciu (2015), risk management theories rely only on the distribution of the tails and the choice of a distribution that underestimates the weight of the extreme values would lead to an underestimated loss.

A review of literature on statistical analysis and modelling of financial time series shows that an assumption that is most commonly made in financial economics theory and applications is that financial returns follow 
the normal distribution. However, imperial studies show that most financial time series data exhibit high kurtosis and skewness. And, as pointed out by Arslan and Genc (2009), when the analysis of real data sets shows that normality assumptions are not tenable, more flexible models that are able to cope with deviation from normality cab be adopted. To accommodate skewness and/or heavy tails in the data, one should model the data with a skewed and/or heavy-tailed distribution (Arslan and Genc, 2009). The authors suggest the use of, amongst other skewed distributions, the skewed generalized t (SGT) originally introduced by Theodossiou (1998). According to Arslan and Genc (2009), the SGT distribution family warrants special attention because it encompasses distributions having both heavy tails and skewness, and many of the widely used distributions such as Student's $t$, normal, Hansen's skew $t$, exponential power, and skew exponential power (SEP) distributions are included as limiting or special cases in the SGT family. The SGT distribution has five parameters (location, scale, skewness and two shape parameters) and provides a flexible tool for modelling data exhibiting diverse levels of tail thickness, , skewness, and peakedness around the location (Theodossiou, 1998). Using stock market indices, exchange rates and the price of gold, Theodossiou (1998) showed that the SGT provides a good fit to the empirical distribution of the data (Liu, Lee and Chang, 2009). As discussed in the preceding paragraph, several authors have used the SGT distribution to model financial time series data. For example, Chu, Nadarajah and Chan (2015) analyse the $\log$ returns of the exchange rate of Bitcoin versus the United States Dollar and fit 15 of the most popular parametric distributions in finance to the log returns, including the Student $t$, skewed $t$ and the generalized $t$. The results of the study show that, based on the BIC, the generalized t distribution ranked number 3 after the generalized hyperbolic and the hyperbolic distributions, in that order. However, to the best of the author's knowledge, no work has been done in the area of modelling the BWP/USD exchange rate using the SGT distribution, and this is the gap that the present investigation attempts to fill.

The objectives of this paper are to empirically establish the baseline characteristics of the daily BWP/USD exchange rate and to identify the most plausible probability distribution for modelling the daily log-returns of the BWP/USD exchange rates amongst four popular members of the skewed generalized $t$ (SGT) family: skewed $t$, skewed generalized error distribution, generalized $t$ and skewed generalized $t$. The results of the study will be of great interest to investors, policy makers, the business community and academics. The rest of the paper is organised as follows. Section 1 presents a description of the data used in the study, the probability models under consideration and the model selection criteria. Section 2 deals with results and their discussions while the last contains the conclusions from the results of the study.

\section{Materials and Methods}

\subsection{Data Description}

The data used in this study consists of daily BWP/USD exchange rates recorded over the period January 2001 to December 2020. The data set was obtained from the Bank of Botswana Exchange rates database (http://www.bankofbotswana.bw/indicators/exchanges). The log return of the exchange rate is calculated as $r_{t}=\ln P_{t}-\ln P_{t-1}$, where $P_{t}$ is the exchange rate at time $\mathrm{t}$, in days. The dataset consists of 4959 daily log returns.

\subsection{SGT Models}

Let $R_{t}$ represent a continuous random variable denoting the log return of the BWP/USD exchange rate at time $\mathrm{t}$, in days, with the probability density function denoted by $f\left(r_{t}\right)$. Then, $R_{t}$ follows one of the following models.

\subsubsection{Skewed generalized $\mathbf{t}$ (SGT)}

The skewed generalized t distribution is a family of continuous probability distributions due to Panayiotis Theodossiou1 (998). It was introduced as a skew extension of the generalized $t$ distribution (see Section 2.2.3 of this article Different parameterizations for the skewed generalized $t$ distribution lead to its various sub-classes discussed in subsequent subsections. The pdf of the SGT has 5 parameters and is given by

$$
f_{S G T}\left(r_{t} ; \mu, \sigma, \lambda, p, q\right)=\frac{p}{2 v \sigma q^{1 / p_{B}}\left(\frac{1}{p^{\prime}}, q\right)\left(\frac{\left|r_{t}-\mu+m\right|}{\left.q(v \sigma)\right|^{p}\left(\lambda \operatorname{sign}\left(r_{t}-\mu+m\right)+1\right)^{p}}+1\right)^{\frac{1}{p}+q}}
$$


where $\mu, \sigma$ and $\lambda$ are the location, scale and skewness parameters and, $\mathrm{p}$ and q measure the kurtosis of the distribution, with $\left\{\left(r_{t}, \mu, \sigma, \lambda, p, q\right):-\infty<r_{t}<\infty,-\infty<\mu<\infty, \sigma>0,-1<\lambda<1, p>0, q>\right.$ $0\}$ and $B(\cdot)$ is the beta function. Further, the mode of the distribution is defined by $m=\frac{2 v \sigma \lambda q^{\frac{1}{p}} B\left(\frac{2}{p^{2}}, q-\frac{1}{p}\right)}{B\left(\frac{1}{p^{2}}, q\right)}$ and $v=q^{-\frac{1}{p}}\left[\left(3 \lambda^{2}+1\right)\left(\frac{B\left(\frac{3}{p}, q-\frac{2}{p}\right)}{B\left(\frac{1}{p}, q\right)}\right)-4 \lambda^{2}\left(\frac{B\left(\frac{2}{p}, q-\frac{1}{p}\right)}{B\left(\frac{1}{p}, q\right)}\right)\right]^{-\frac{1}{2}}$.

\subsubsection{Skewed Generalized error distribution (SGED)}

The skewed generalized error distribution was developed by Theodossiou, P. (1998).to fit the empirical distribution of log returns of a number of some well-known financial assets at various data frequencies. Its pdf is given by

$$
f_{S G T}\left(r_{t} ; \mu, \sigma, \lambda, p, q=\infty\right)=\frac{\left.p e^{-\left(\frac{\left|r_{t}-\mu+m\right|}{v \sigma\left(1+\lambda s i g n\left(r_{t}-\mu+m\right)\right.}\right)}\right)^{p}}{2 v \sigma \Gamma(1 / p)}
$$

where $\left\{\left(r_{t}, \mu, \sigma, \lambda, p, q\right):-\infty<r_{t}<\infty,-\infty<\mu<\infty, \sigma>0,-1<\lambda<1, p>0\right\}$, mode $m=\frac{2 \frac{2}{p} v \sigma \lambda \Gamma\left(\frac{1}{2}+\frac{1}{p}\right)}{\sqrt{\pi}}$ and

$v=\left[\frac{\pi\left(1+3 \lambda^{2}\right) \Gamma\left(\frac{3}{p}\right)-16^{\frac{1}{p}} \lambda^{2} \Gamma\left(\frac{1}{2}+\frac{1}{p}\right)^{2} \Gamma\left(\frac{1}{p}\right)}{\pi \Gamma\left(\frac{1}{p}\right)}\right]^{-\frac{1}{2}}$.

\subsubsection{Generalized $\mathbf{t}$ (GT) distribution}

This distribution was first proposed by McDonald and Newey (1988) to apply a partially adaptive estimation of regression models. Its pdf is given by

$$
f_{S G T}\left(r_{t} ; \mu, \sigma, \lambda=0, p, q\right)=\frac{p}{2 v \sigma q^{1 / p_{B}}\left(\frac{1}{p^{\prime}} q\right)\left(\frac{\left|r_{t}-\mu+m\right|^{p}}{q(v \sigma)^{p}}+1\right)^{1 / p+q}}
$$

where $\left\{\left(r_{t}, \mu, \sigma, p, q\right):-\infty<r_{t}<\infty,-\infty<\mu<\infty, \sigma>0, p>0, q>0\right\}$ and $v=\frac{1}{q^{1 / p}}\left(\frac{B\left(\frac{1}{p}, q\right)}{B(3 / p, q-2 / p)}\right)^{1 / 2}$.

\subsubsection{Skewed $\mathbf{t}$ (ST) distribution}

The skewed t distribution, introduced by Hansen (1994), is an extension of the well-known symmetric Student-t distribution, with a pdf of the form

$f_{S G T}\left(r_{t} ; \mu, \sigma, \lambda, p=2, q\right)=\frac{\Gamma(1 / 2+q)}{v \sigma(\pi q)^{1 / 2} \Gamma(q)\left(\frac{\left|r_{t}-\mu+m\right|^{p}}{q(v \sigma)^{2}\left(\lambda \operatorname{sign}\left(r_{t}-\mu+m\right)+1\right)^{2}}+1\right)^{1 / 2+q}}$

where $m=\frac{2 v \sigma \lambda q^{1 / 2} \Gamma(q-1 / 2)}{\pi^{1 / 2} \Gamma(q+1 / 2)}$ and $v=q^{-1 / 2}\left[\left(3 \lambda^{2}+1\right)\left(\frac{1}{2 q-2}\right)-\frac{4 \lambda^{2}}{\pi}\left(\frac{\Gamma(q-1 / 2)}{\Gamma(q)}\right)^{2}\right]^{-1 / 2}$.

\subsection{Parameter Estimation}

Consider fitting an SGT $(\mu, \sigma, \lambda, p, q)$ distribution with a probability density function in Equation (1) to the daily $\log$ returns $r_{1}, r_{2}, \cdots, r_{n} \in \mathbb{R}$, where $\mathrm{n}$ is the total number of days, using the maximum likelihood estimation (MLE) method. Arslan and Genc (2009) give (in Equation (18)), the log-likelihood function of the SGT $(\mu, \sigma, \lambda, p, q)$, for a generic data set $x_{1}, x_{2}, \cdots, x_{n} \in \mathbb{R}$, as stated in Equation (4)

$$
l(\mu, \sigma, \lambda, p, q)=n \log \frac{p}{2 B\left(\frac{1}{p^{\prime}}, q\right) q^{1 / p}}-n \log \sigma-\left(\frac{p q+1}{p}\right) \sum_{i=1}^{n} \log \left(1+\frac{\left|x_{i}-\mu\right|^{p}}{\left[1+\operatorname{sign}\left(x_{i}-\mu\right) \lambda\right]^{p} q \sigma^{p}}\right)
$$

The MLEs of the parameters $\mu, \sigma, \lambda, p$ and $q$ are obtained by maximising the log-likelihood function [4]. This involves taking the derivative of Equation [4] with respect to each parameter and setting the result to zero to obtain the likelihood equations stated in Equations (19)-(23) of Arslan and Genc (2009). These nonlinear likelihood equations cannot be readily solved analytically. Therefore, the MLEs for the parameters of the SGT $(\mu, \sigma, \lambda, p, q)$ distribution must be obtained using numerical techniques. In the present study, we find the MLEs of $\mu, \sigma, \lambda, p$ and $q$ by using the R Package sgt with the nlminb maximisation algorithm. 


\subsection{Model Selection Criteria}

Model selection is based on two widely used information theoretic criteria, namely, the Akaike information criterion (AIC) and Bayesian information criterion (BIC), where the

and

$$
A I C=2 k-2 \ln l(\widehat{\boldsymbol{\theta}})
$$

$$
B I C=k \ln n-2 \ln l(\widehat{\boldsymbol{\theta}})
$$

where $\widehat{\boldsymbol{\theta}}=(\hat{\mu}, \hat{\sigma}, \hat{\lambda}, \hat{p}, \widehat{q}), l(\widehat{\boldsymbol{\theta}})$ is the value of the likelihood evaluated at the vector of parameter estimates $\widehat{\boldsymbol{\theta}}, k$ is the number of parameters of the model and $n$ is the sample size.

In addition, standard errors and the $t$ test are used to assess the significance of each parameter in a given model.

\section{Results and Analysis}

\subsection{Preliminary Analysis}

Figure 1 shows historical nominal exchange rates between the Botswana Pula and the US Dollar between $01 / 01 / 2001$ and $24 / 12 / 2020$. From the figure it is clear that there was some volatility in the trend of the BWP/USD exchange rate from about 2001 to 2005, followed by another bout of instability around 20082010. These could be attributed to the economic instability experienced in world diamond markets during

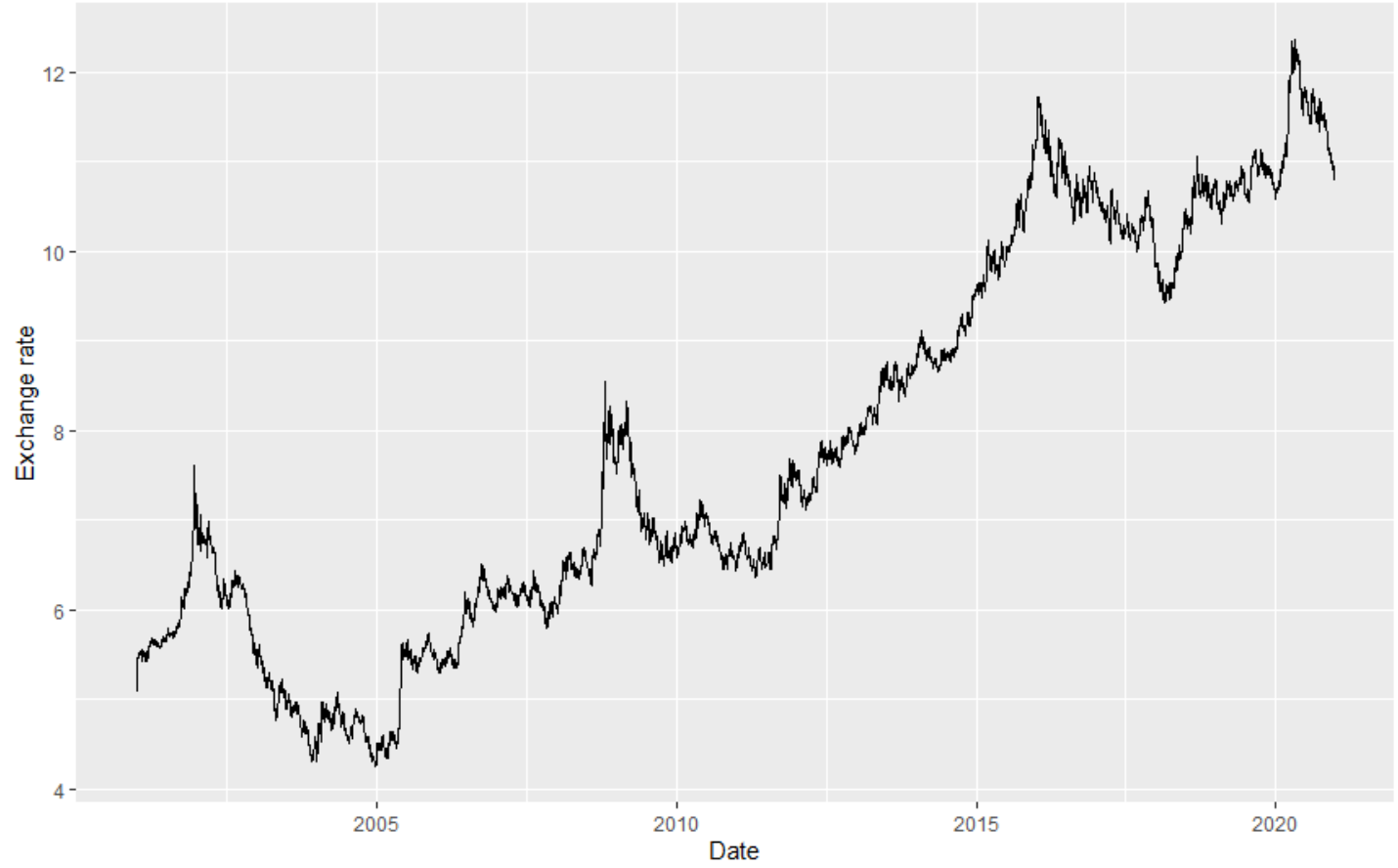

Figure 1: Time series plot of the BWP/USD exchange rates from January 1, 2001 to December 24, 2020

those periods, among other factors. Thereafter, the nominal BWP/USD exchange rate increased steadily with minimal variations until about 2017, after which series exhibited some degree of volatility. Overall, however, the time series plot exhibits non-stationary variations in the BWP/USD exchange rate over the sample period. And, to achieve stationarity in the series, we take log returns of the historical exchange rates. The log returns are plotted in Figure 2. From this figure, Summary statistics of the daily BWP/USD log returns are recorded in Table 1. And, as expected with financial time series data, the log returns have approximately a mean of zero, are negatively skewed and have a very high Kurtosis of 26.8772 , which is higher than the gold standard value of 3 for the normal distribution. The Jarque-Bera test for normality also rejects the null hypothesis of normality of the daily BWP/USD exchange rate returns. 


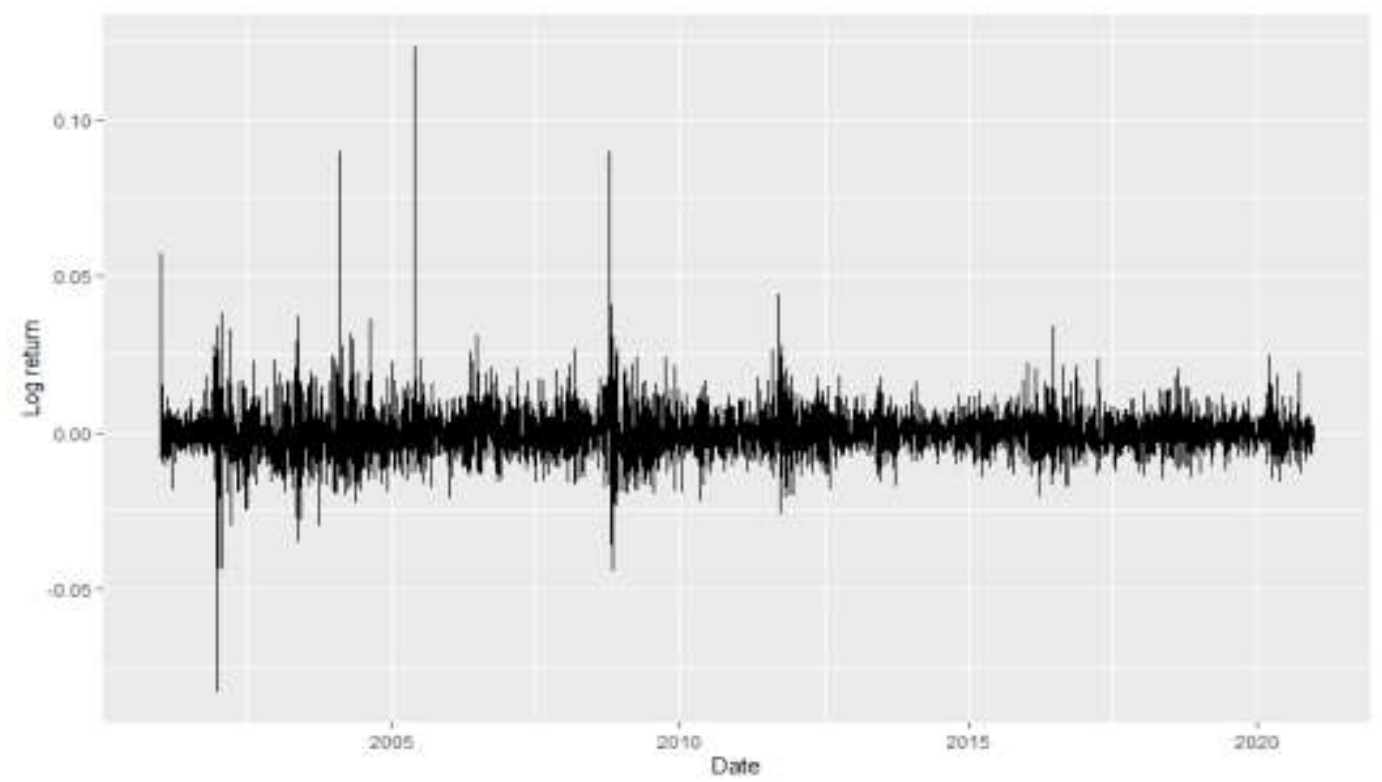

Figure 2: Time series plot of the returns of the BWP/USD exchange rates from January 1, 2001 to December 24, 2020
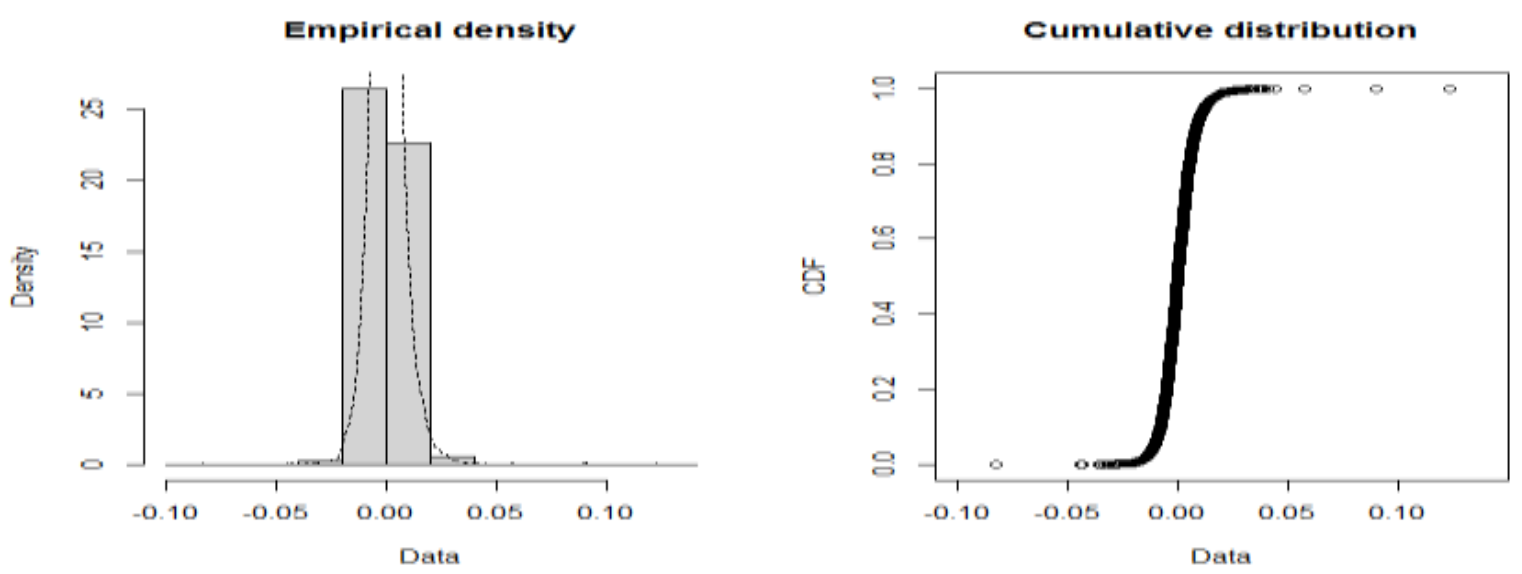

Figure 3: Hi from January 1, 2001 to December 24, 2020 histogram and Cumulative distribution function of the empirical distribution of the BWP/USD exchange rate returns, January 1, 2001 to December 24, 2020

Table 1. Some summary statistics of the log returns of the BWP/USD

\begin{tabular}{|l|l|}
\hline Measure & Value \\
\hline Mean & 0.000152 \\
\hline Standard Error & 0.0001 \\
\hline Median & 0 \\
\hline Standard Deviation & 0.007811 \\
\hline Kurtosis & 26.8772 \\
\hline Skewness & 1.450944 \\
\hline Range & 0.205581 \\
\hline Minimum & -0.082498 \\
\hline Maximum & 0.123083 \\
\hline Count & 4959 \\
\hline Jargue-Bera statistic & 119291 (p-value < 2.2e-16) \\
\hline
\end{tabular}

\subsection{Fitting the Models}

The MLE estimates of the parameters of the three models considered in this investigation are given in Figure 4: skewed t (Fig. 4a), skewed generalized error (Fig. 4b), generalized t (Fig.4c) and skewed generalized $t$ (Fig. 4d). The p-values of the $t$ test statistics associated with all the parameters are all equal to zero, except 
in the case of the location parameter $\mu$ of the skewed $\mathrm{t}$ and the skewed generalized error distributions. These very small $\mathrm{p}$-values indicate overwhelming evidence for the inclusion of the associated parameters in the respective models.

Table 2 contains the Log-likelihoods and the two model selection criteria for the fitted distributions. It is very clear from the table that, based on the values of both the AIC and BIC, the model that gives the best fit is the skewed generalized error distribution. The extended model, the Skewed generalized $t$, gives the worst fit based on both the two criteria.

Table 2 Log-likelihoods and the two model selection criteria for the fitted distributions

\begin{tabular}{|l|c|c|c|c|c|}
\hline Model & No. parameters k & Loglikehood & AIC & BIC & Rank \\
\hline Skewed t & 4 & 17561.71 & -35115.4 & -35089.4 & 1 \\
\hline Skewed generalized error & 4 & 17480.92 & -34953.8 & -34927.8 & 2 \\
\hline Generalized t & 4 & 14522.92 & -29037.8 & -29011.8 & 4 \\
\hline Skewed generalized t & 5 & 15892.70 & -31775.4 & -31742.9 & 3 \\
\hline
\end{tabular}

\section{Conclusions}

In this paper, historical daily log returns of the Botswana Pula and United Sates of America Dollar are analysed and modelled using the skewed generalized $t$ distribution and three of its related distributions, namely, the skewed $t$, skewed generalized error and generalized t. The 5-parameter skewed generalized $t$ is one of the most flexible family of distributions and popular models for modelling financial returns series. We find that the daily returns of the BWP/USD exchange rates exhibit stylised characteristics empirically associated with financial returns data: they have a mean of approximately zero, are negatively moderately skewed and highly leptokurtic. Furthermore, based on the values of both the AIC and BIC, the model that gives the best fit to the series of returns is the skewed t distribution, which is revealed in the literature as one of the very popular models for fitting financial time series. The skewed generalized error distribution, another popular model for modelling financial time series, ranks a close second to the skewed $t$ and performs better than the generalized $t$ based on the two information theoretic model selection criteria. The five-parameter skewed generalized $t$ gives the worst fit to the series of daily BWP/USD returns.

Subsequent work in this important empirical research will attempt to address the crucial issue of deriving measures for forecasting risk for the BWP/USD nominal exchange rate returns, especially the value at risk (VaR). It will also be of interest to stakeholders for the research to investigate if varying the frequency of the data (weekly, monthly or annually) will lead to different results on the appropriateness of the probability distributions under consideration.

\section{Ethics approval and consent to participate}

Not applicable.

\section{Data Availability}

Readers can access the data on the daily BWP/USD exchange rate underlying the findings of this study from the Bank of Botswana Exchange rates database (http://www.bankofbotswana.bw/indicators/exchanges).

\section{Conflicts of Interest}

The author declares that there is no conflict of interest regarding the publication of this paper.

\section{Funding Statement}

Not applicable.

\section{Authors' contributions}

$\mathrm{WM}$ is the single author of this article. 


\section{References}

1. African Development Bank (2016). Botswana's Mineral Revenues, Expenditure and Savings Policy Case Study. African Natural Resources Center, African Development Bank, Abidjan 01, Côte d'Ivoir.

2. Arslan and Genc (2009). The skew generalized t (SGT) distribution as the scale mixture of a skew exponential power distribution and its applications in robust estimation. Statistics, 43 (5), 481-498.

3. Baciu. I. (2015). Stochastic models for forecasting inflation rate: Empirical evidence from Romania, 7th International Conference on Globalization and Higher Education in Economics and Business Administration - GEBA - 2013, Elsevier - Procedia.

4. Chu, J., Nadarajah, S. and Chan, S. (2015). Statistical Analysis of the Exchange Rate of Bitcoin. PLos ONE, 10 (7): e0133678.doi.10.1371/journal.pone.0133678.

5. Demarta, S. and McNeil, A. (2005). The t copula and related copulas. Int. Statist. Rev. 73, 111-129.

6. Hansen, B. E. (1994). Autoregressive Conditional Density Estimation. International Economic Review 35 (3), 705-730.

7. Hung-Chun Liu, Ming-Chih Lee and Ching-Mo Chang (2009). The role of the SGT distribution in Value-at-Risk estimation: evidence from the WTI crude oil market. Investment Management and Financial Innovations, 6 (1).

8. McDonald, J.B., Newey, W.K (1988). Partially Adaptive Estimation of Regression Models via the Generalized t Distribution. Econom. Theory, 4, 428-457.

9. Sejoe, S., Narain, S. and Kahaka, Z. (2020). Purchasing Power Parity Theory for Pula/Rand and BWP/USD Exchange Rates in Botswana, 2020-3704-AJBE.

10. Theodossiou, P. (2015). Financial data and the skewed generalized t distribution. Multinational Finance Journal, 19 (4), 223-266.

11. Theodossiou, P. (1998). Financial data and the skewed generalized t distribution. Management Science, 44 (12), Part 1 of 2, 1650-1661. 